\begin{tabular}{|l|l|l|}
\hline \multicolumn{2}{|c|}{ PublisherInfo } \\
\hline \hline PublisherName & $:$ & BioMed Central \\
\hline \hline PublisherLocation & $:$ & London \\
\hline \hline PublisherImprintName & $:$ & BioMed Central \\
\hline \hline
\end{tabular}

\title{
Virtual restriction analysis
}

\begin{tabular}{|l|l|l||}
\hline \multicolumn{2}{|c|}{ ArticleInfo } \\
\hline \hline ArticleID & $:$ & 3895 \\
\hline \hline ArticleDOI & $:$ & $10.1186 /$ gb-2001-2-11-reports0042 \\
\hline \hline ArticleCitationID & $:$ & reports0042 \\
\hline \hline ArticleSequenceNumber & $:$ & 20 \\
\hline \hline ArticleCategory & $:$ & Paper report \\
\hline \hline ArticleFirstPage & $:$ & 1 \\
\hline \hline ArticleLastPage & $:$ & 4 \\
\hline \hline & & RegistrationDate $: 2001-8-20$ \\
ArticleHistory & $:$ & Received $\quad: \quad 2001-8-20$ \\
& $:$ & OnlineDate $\quad$ 2001-10-26 \\
\hline \hline ArticleCopyright & $:$ & BioMed Central Ltd2001 \\
\hline \hline ArticleGrants & $:$ & \\
\hline \hline
\end{tabular}




\begin{tabular}{|l|l|l|}
\hline ArticleContext & $:$ & 13059221111 \\
\hline
\end{tabular}

\section{Agnieszka M Lichanska}

\section{Abstract}

A new computational method for whole-genome analysis by in silico restriction mapping has been developed and has been evaluated by comparison with experimental restriction analysis of tumor DNA.

\section{Significance and context}

The accumulation of genomic DNA sequences has allowed the development of in silico computational tools for analyzing the genome. Most of these methods are predictive, and there is a great need for tools to help analyze results obtained by new experimental techniques. One such experimental technique is restriction landmark genome scanning (RLGS), first described a decade ago, which allows thousands of restriction fragments to be separated and visualized simultaneously. A lack of any rapid way of identifying the DNA fragments in the scans proved an obstacle to its wide use, however. Rouillard et al. have now developed a computational method, Virtual Genome Scan (VGS), that yields virtual scans of the human genome using in silico restriction-enzyme mapping of genomic sequences from the databases.

\section{Key results}

VGS is a novel computational method designed to analyze genome restriction-mapping data predicted from genome sequence. The computed DNA fragments from an in silico restriction scan were superimposed on the images of gels obtained by experimental RLGS using the same restriction enzymes (see Methodological innovations for details). The authors first directly compared the predictive power of VGS with experimental RLGS of neuroblastoma DNA, followed by fragment sequencing. VGS predicted fragments of the right size and correct sequence; the only ones missing from the predicted results were absent because of a lack of sequence data in the database. The usefulness of the method for analyzing tumor tissue was highlighted by a second experiment, in which Rouillard et al. showed that some predicted fragments did not appear on the RLGS gels as a result of methylation in the tumor DNA.

A third experiment analyzed a $<230$ kilobase $(\mathrm{kb})$ region amplified on chromosome $17 \mathrm{q} 23$ in a breast tumor. The RLGS pattern showed two highly amplified fragments. VGS predicted the two amplified fragments and indicated that there should also be two more fragments that are above the size limit of the 
first-dimension gel used for the RLGS. VGS not only predicted these fragments but also allowed identification of the genes they contain. The expression of these genes in tumor and control samples could then be rapidly analyzed by PCR.

\section{Methodological innovations}

Rouillard et al. used finished and unfinished human genome sequence from GenBank for analysis, with unfinished sequence split into fragments to avoid any incorrectly joined sequences. On the basis of this sequence information, the sizes of the NotI-NotI or NotI-EcoRV genomic fragments were computed. Fragments generated in the in silico NotI digests were further analyzed to compute the sizes of the fragments generated by subsequent HinfI or DpnII digests. Overlapping clones were then removed, resulting in 4,840 fragments for Hinfl and 5,210 fragments for DpnII. These fragments form the basis of the VGS database.

\section{Links}

More about this method is available at Virtual Genome Scan Server.

\section{Conclusions}

Rouillard et al. have devised a method for predicting the genomic fragments on RLGS gels by an in silico restriction scan. Comparison of the experimental and computed fragment patterns shows that VGS is useful for analyzing RLGS data. It can predict genomic fragments independently of their methylation status and provides information on fragment DNA sequence, allowing rapid identification of genes within a fragment of interest.

\section{Reporter's comments}

This is a major breakthrough in genome analysis using restriction mapping. Combining an experimental method with a predictive tool such as VGS overcomes some of the difficulties in the analysis of restriction landmark genome scans. It should be stressed that the method is not a solution for all the problems, especially because the in silico prediction of fragments is limited by the availability of finished sequence. VGS has the potential to improve as new sequence becomes available, however. The main advantage of the method is that it can be used to study any genome, not just human. 
There are some potential problems in relating VGS prediction results to the RLGS results, because of discrepancies between predicted and observed fragment migration. Some differences are informative, for example those due to DNA methylation; others are related to problems with gels, for example aberrant DNA migration and the resolution limit of the gel leads to the absence of some fragments in the experimental scan.

\section{Table of links}

Genome Research

Virtual Genome Scan Server

\section{References}

1. Rouillard JM, Erson AE, Kuick R, Asakawa J, Wimmer K, Muleris M, Petty EM, Hanash S: Virtual Genome Scan: a tool for restriction landmark-based scanning of the human genome. Genome Res. 2001, 11: 1453-1459. 1088-9051 